\title{
A NON-INTERRUPTING ELECTRON BEAM DIAGNOSTIC USING COHERENT OFF-AXIS UNDULATOR RADIATION
}

\author{
C.P. Neuman", W.S. Graves, Brookhaven National Laboratory, Upton, NY \\ P.G. O'Shea, University of Maryland, College Park, MD
}

\section{Abstract}

We propose a technique for measuring the length of an electron bunch from a linear accelerator without interrupting the electron beam. Bunch lengths are measured by observing off-axis undulator radiation. The wavelength of undulator radiation increases with the angle of emission. At angles as large as 10 degrees, the wavelength may be longer than the electron bunch, and as a result coherence effects emerge. As the angle of observation increases and the radiation becomes coherent, the intensity may change by up to a factor of $10^{9}$. The angle at which this change occurs is related to the bunch length. Thus the bunch length may be obtained by observing the change in intensity of the radiation with respect to the angle of observation. This electron beam diagnostic does not interrupt the electron beam and would be useful for single-pass FELs. Electron bunches could be characterized while the FEL is operating, and thus the FEL could be optimized in real time. In this paper, we develop the theory of coherent off-axis undulator radiation (COUR), including near-field effects, and we discuss future experimental efforts to observe this radiation and to use it to characterize electron bunches.

\section{THEORY}

\subsection{Introduction}

In most descriptions of undulator radiation, the discussion is limited to small angles of observation, particularly angles less than $\frac{1}{\gamma \sqrt{N_{w}}}$ radians [1], where $N_{w}$ is the number of periods in the undulator. In our study we explore the radiation outside the narrow $\frac{1}{\gamma \sqrt{N_{w}}}$ cone.

The peak wavelength of undulator radiation increases with angle:

$$
\lambda_{0}(\theta)=\frac{\lambda_{w}}{2 \gamma^{2}}\left[\left(1+a_{w}{ }^{2}\right) \cos \theta+2 \gamma^{2}(1-\cos \theta)\right],
$$

where $\theta$ is the angle of observation, $\lambda_{w}$ is the undulator period, $a_{w}$ is the undulator parameter, and $\gamma$ is the relativistic factor of the electron beam. Typical values of $\lambda_{0}$, for $\lambda_{w}=4 \mathrm{~cm}$ and $\gamma=400$, are $250 \mathrm{~nm}$ on-axis, and 1.3 $\mathrm{mm}$ for $\theta=15^{\circ}$. Note that for $\gamma$ large and $\theta$ greater than a few degrees, Eq. 1 becomes

$$
\lambda_{0}(\theta) \approx \lambda_{w}(1-\cos \theta)
$$

\footnotetext{
*Corresponding author. Neuman@bnl.gov.
}

which is independent of the electron beam energy.

For small angles, the radiation typically has a wavelength that is much shorter than a bunch length. Here, the electrons radiate incoherently, and the intensity of the radiation scales linearly with the number of electrons. For a large enough angle, the wavelength may be much larger than the bunch length. In this case, the electrons radiate coherently, and the intensity of the radiation scales with the square of the number of electrons. As the angle of observation is increased and the radiation becomes coherent, the intensity will jump by a factor equal to the number of electrons, which is typically on the order of $10^{9}$. The angle at which this large jump occurs depends on the electron bunch length. Thus, relative bunch length measurements may be performed by observing undulator radiation from a range of angles [2].

\subsection{Energy Calculations for Single Electron}

We calculate the total energy and the spectral energy of the emitted undulator radiation using a straightforward approach derived from Liénard-Wiechert potentials [3]. The geometry for the calculation is shown in Fig. 1.

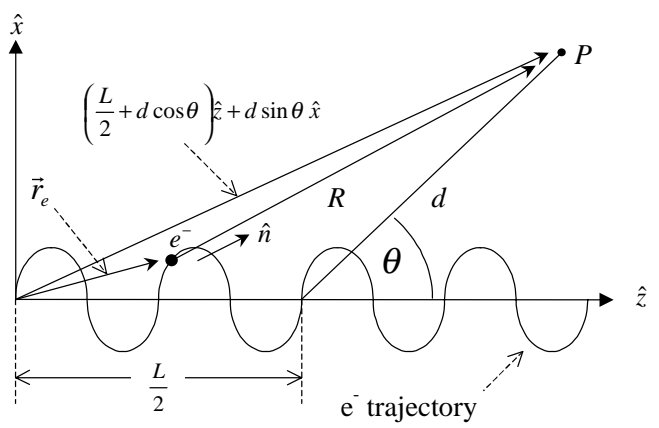

Figure 1: Geometry for energy calculations. $P$ is the point of observation, $\vec{r}_{e}$ is the instantaneous position of the electron, $R$ and $\hat{n}$ are the distance and direction, respectively, from the electron to the point of observation, and $d$ is the distance from the center of the undulator to the point of observation.

The total power per unit solid angle radiated by an accelerating electron, in the electron's time, is given by

$$
\frac{d P\left(t^{\prime}\right)}{d \Omega}=R^{2} \vec{S} \cdot \hat{n}(1-\hat{n} \cdot \vec{\beta})=c \varepsilon_{0} E^{2} R^{2}(1-\hat{n} \cdot \vec{\beta}),
$$

where $\vec{S}$ is the Poynting vector associated with the radiation. The total energy per unit solid angle is obtained 
by integrating over time. The electric field $\vec{E}$ can be derived from Liénard-Wiechert potentials:

$$
\vec{E}=\frac{q^{2}}{4 \pi \varepsilon_{0}}\left\{\frac{1}{\gamma^{2} R^{2}} \frac{\hat{n}-\vec{\beta}}{(1-\hat{n}-\vec{\beta})^{3}}+\frac{1}{c R} \frac{\hat{n} \times[(\hat{n}-\vec{\beta}) \times \dot{\vec{\beta}}]}{(1-\hat{n}-\vec{\beta})^{3}}\right\}
$$

The first term represents the velocity field, which does not radiate and is thus disregarded in this study. The second term represents the radiating acceleration field and depends on both the velocity and acceleration of the electron. Thus, the energy per unit solid angle is:

$$
\frac{d W}{d \Omega}=\frac{1}{4 \pi \varepsilon_{0}} \frac{q^{2}}{4 \pi c} \int_{0}^{\frac{L}{\bar{\beta}_{c} c}} \frac{\hat{n} \times\left.[(\hat{n}-\vec{\beta}) \times \dot{\vec{\beta}}]\right|^{2}}{(1-\hat{n} \cdot \vec{\beta})^{5}} d t
$$

To calculate the energy spectrum, we begin with an expression for the energy per unit solid angle:

$$
\frac{d W}{d \Omega}=\int_{-\infty}^{\infty}|\vec{A}(t)|^{2} d t=\int_{-\infty}^{\infty}|\vec{A}(\omega)|^{2} d \omega
$$

where $\vec{A}(t)$ is defined as

$$
\vec{A}(t) \equiv \sqrt{\varepsilon_{0} c} R \vec{E},
$$

and $\vec{A}(\omega)$ is the Fourier Transform of $\vec{A}(t)$ :

$$
\vec{A}(\omega)=\frac{1}{\sqrt{2 \pi}} \int_{-\infty}^{\infty} \vec{A}(t) e^{i \omega t} d t
$$

The total energy per unit solid angle can be expressed as

$$
\frac{d W}{d \Omega}=\int_{0}^{\infty} \frac{d^{2} W}{d \omega d \Omega} d \omega
$$

where

$$
\frac{d^{2} W}{d \omega d \Omega} \equiv 2|\vec{A}(\omega)|^{2}
$$

is the energy radiated per unit solid angle per unit angular frequency. Using Eq. 2, the spectral energy becomes:

$$
\frac{d^{2} W}{d \omega d \Omega}=\frac{\varepsilon_{0}}{c \pi} \frac{q^{2}}{\left(4 \pi \varepsilon_{0}\right)^{2}}\left|\int_{0}^{\frac{L}{\bar{\beta}_{c} c}} e^{i \omega\left(t+\frac{R}{c}\right)} \frac{\hat{n} \times[(\hat{n}-\vec{\beta}) \times \dot{\vec{\beta}}]}{(1-\hat{n} \cdot \vec{\beta})^{2}} d t\right|^{2}
$$

The total energy and spectral energy are related to the respective energies per unit solid angle by

$$
\begin{aligned}
& W=\frac{1}{d^{2}}\left(\frac{d W}{d \Omega}\right) \Delta A \\
& \frac{d W}{d \omega}=\frac{1}{d^{2}}\left(\frac{d^{2} W}{d \omega d \Omega}\right) \Delta A
\end{aligned}
$$

where $d$ is the distance of the detector from the center of the undulator, and $\Delta A$ is the area of the detector entrance aperture.

To evaluate Eqs. 3 and 4, the normalized acceleration and velocity of the electron are needed, along with the approximation $z \approx \bar{\beta}_{z} c t$. These are calculated by applying the Lorentz force equation to the periodic undulator field, yielding the following well-known expressions:

$$
\begin{aligned}
& \dot{\beta}_{x}=\frac{-a_{w} \sqrt{2}}{\gamma} \sin \left(k_{w} z\right) k_{w} \bar{\beta}_{z} c \\
& \dot{\beta}_{z}=\frac{a_{w}{ }^{2}}{\gamma^{2}} \sin \left(2 k_{w} z\right) k_{w} \bar{\beta}_{z} c \\
& \beta_{x}=\frac{a_{w} \sqrt{2}}{\gamma} \cos \left(k_{w} z\right) \\
& \beta_{z}=\bar{\beta}_{z}-\frac{a_{w}{ }^{2}}{2 \gamma^{2}} \cos \left(2 k_{w} z\right), \bar{\beta}_{z} \equiv 1-\frac{1}{2 \gamma^{2}}\left(1+{a_{w}}^{2}\right)
\end{aligned}
$$

\subsection{Bunch Form Factor for $N_{e}$ Electrons}

The energy for $N_{e}$ electrons is related to the energy for a single electron by [4]:

$$
W_{N_{e} \text { electrons }}=W_{\text {1electron }}\left[N_{e}+N_{e}\left(N_{e}-1\right) f(\omega)\right] \text {, }
$$

where $f(\omega)$ is the bunch form factor:

$$
f(\omega)=\left|\iint d y d z S_{y}(y) S_{z}(z) e^{-i \frac{\omega}{c} y \sin \theta+i \frac{\omega}{c} z \cos \theta}\right|^{2}
$$

$S_{y}(y)$ and $S_{z}(z)$ are the transverse and longitudinal electron bunch densities, respectively. In this study we use Gaussian-shaped bunch densities. Eqs. 8 and 9 include the coherence effects described above: for $\omega$ large $f(\omega) \approx 0$, and $W \propto N_{e}$; for $\omega$ small $f(\omega) \approx 1$, and $W \propto N_{e}^{2}$.

The form factor depends on both the length and width of the electron bunch. In this study we only consider the effects of the bunch length since the length is a critical quantity in many beam physics and FEL applications.

\subsection{Near-Field Effects}

Near-field effects are introduced by accounting for the fact that $\hat{n}$ and $R$ change as the electron passes through the undulator (see Fig. 1). The vector $R \hat{n}$ can be expressed as

$$
R(t) \hat{n}(t)=\left(\frac{L}{2}+d \cos \theta\right) \hat{z}+d \sin \theta \hat{x}-\vec{r}_{e}(t),
$$

where $\vec{r}_{e}(t)$ is the electron trajectory. $\vec{r}_{e}$ is calculated by integrating Eqs. 7 with the approximation $z \approx \bar{\beta}_{z} c t$ :

$$
\begin{aligned}
& r_{x}=\frac{a_{w} \sqrt{2}}{\gamma k_{w} \bar{\beta}_{z}} \sin \left(k_{w} \bar{\beta}_{z} c t\right) \\
& r_{z}=\bar{\beta}_{z} c t-\frac{a_{w}{ }^{2}}{4 \gamma^{2} k_{w} \bar{\beta}_{z}} \sin \left(2 k_{w} \bar{\beta}_{z} c t\right)
\end{aligned}
$$

Thus, $R(t)$ is obtained by taking the magnitude of Eq. 10, and $\hat{n}(t)$ by dividing Eq. 10 by $R(t)$. The consequences of including near-field effects are discussed below.

\subsection{Results}

Eqs. 3 and 4 are evaluated numerically. A plot of the total energy radiated versus angle of emission is displayed in Fig. 2. The two traces correspond to two different bunch lengths. It can be seen that the bunch length determines 
the angle at which the intensity of the radiation increases dramatically. This characteristic of COUR may allow us to measure relative bunch lengths by observing COUR at a range of angles.

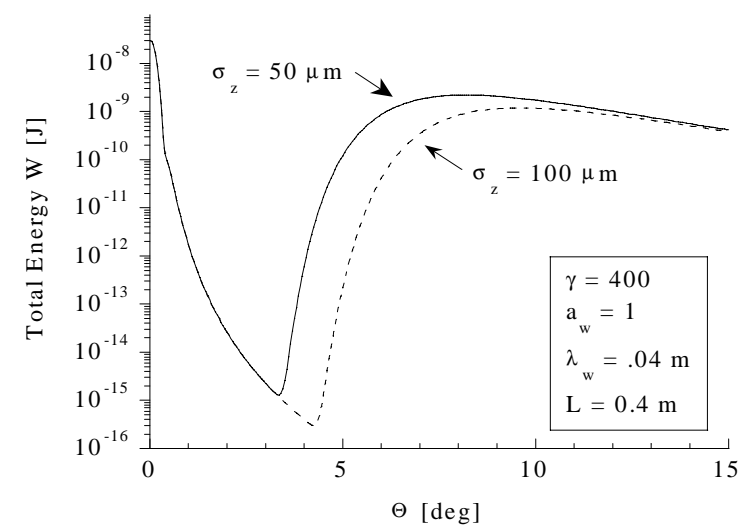

Figure 2: The effect of bunch length on COUR. $\sigma_{\mathrm{z}}$ is the rms bunch length divided by $\sqrt{2}$, as defined in [4].

Calculations reveal that near-field effects have a significant effect on the energy spectrum for detector distances of less than $1 \mathrm{~m}$ (see Fig. 3). The fundamental peak is shifted to higher frequencies and is broadened. Without near-field effects, the spectrum displays the expected $\operatorname{sinc}^{2}\left(\mathrm{~N}_{\mathrm{w}} \pi \frac{\Delta \omega}{\omega_{0}}\right)$ behavior on-axis, and slight variations of this behavior off-axis, as seen in Fig. 3. This behavior is disturbed in the near-field calculation. As the detector distance becomes large, greater than $1 \mathrm{~m}$ in this case, the near-field and far-field calculations begin to have similar behavior.

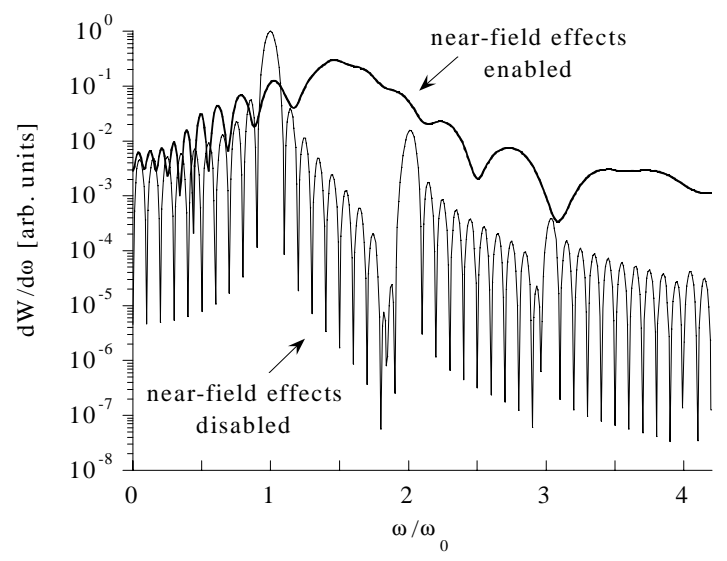

Figure 3: Effect of near-field effects on energy spectrum for a single electron. $\theta=5^{\circ}$, detector distance $d=.5 \mathrm{~m}$. All other parameters are the same as in Fig. 2.

\section{EXPERIMENT}

The goals of a COUR experiment are to observe COUR and to measure relative bunch lengths using COUR.

A COUR experiment is planned for the DUV-FEL facility, a new priority for BNL designed to produce a coherent light source in the ultraviolet range [5]. The linear accelerator will produce $210 \mathrm{MeV}$ electrons. The undulator for the COUR experiment is a 13 period prototype of the $10 \mathrm{~m}$ NISUS undulator, now installed at the DUV-FEL. The NISUS prototype has an undulator parameter of up to 1.44 and an undulator period of 3.89 $\mathrm{cm}$ [6].

A liquid helium-cooled bolometer detector will be used to detect energy levels as low as $10^{-12} \mathrm{~J}$. A spectrometer will be used to determine the spectral content of the radiation. Radiation will exit the beamline through an aperture with a diamond window, after being reflected away from the beamline by a small mirror (see Fig. 4).

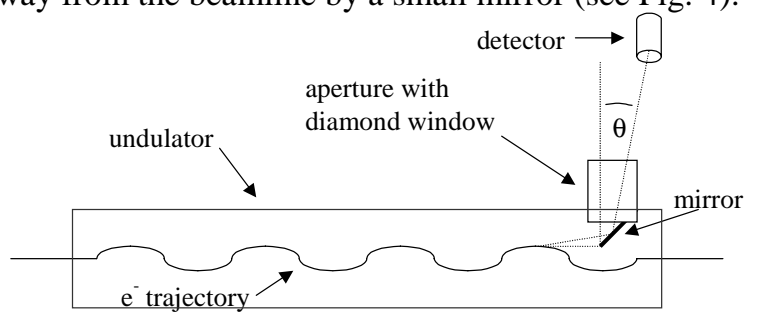

Figure 4: COUR experiment.

The COUR experiment is one of a series of beam diagnostics experiments planned for the DUV-FEL [7]. The various diagnostic techniques will be compared and will be used in conjunction with each other.

\section{CONCLUSION}

We have shown that a promising technique for measuring electron bunch lengths is to observe coherent off-axis undulator radiation (COUR). Our technique is noninterrupting and thus will be useful for single-pass FELs, where bunch lengths could be measured while an FEL is operating. Calculations including near-field effects show the expected energy levels and energy spectra. An experiment at the DUV-FEL facility at BNL will verify the calculations and will demonstrate the use of COUR to measure electron bunch lengths.

\section{ACKNOWLEDGEMENTS}

The authors wish to thank Larry Carr and Jim Murphy of BNL for helpful discussions on detection and electromagnetic theory, respectively. Computational support, in the form of Cray computer time, was provided by the North Carolina Supercomputing Center. This work was supported, in part, by a U.S. Army AASERT grant to the Duke University FEL Lab.

\section{REFERENCES}

[1] S. Krinsky et al, from E.E. Koch, ed., Handbook on Synchrotron Radiation. Amsterdam, North Holland, 1983, 152.

[2] C.P. Neuman et al, Proc. of FEL98 Conference.

[3] J.D. Jackson, Classical Electrodynamics. New York: Wiley, 1975, 662-70.

[4] E.B. Blum et al, Nucl. Instr. and Meth. A 307 (1991), 568-76.

[5] I. Ben-Zvi et al, Proc. of SPIE, 2988 (1997), 15-19.

[6] D.C. Quimby et al, Nucl. Instr. and Meth. A 285 (1989), 281.

[7] W.S. Graves et al, Proc. of IEEE PAC99. 